\title{
Investigation of fracture zone properties using reflected seismic waves from passive microseismicity
}

\author{
RA Lynch Institute of Mine Seismology, Australia
}

\begin{abstract}
A new method has been developed for imaging seismic reflectors using seismogram data recorded by a few standard triaxial geophones. A directional migration approach reduces imaging artefacts due to the low aperture of the sensor array. This is possible by measuring the polarisation in the seismogram coda and considering all four reflected body-waves: $P P^{\prime}, P S^{\prime}, S P^{\prime}$ and SS'.

This technique has been applied to seismograms recorded by deep borehole sensors at Williams mine, Canada, in order to invert for fracture zone properties surrounding the open pit. A fracture zone thickness of $20 \mathrm{~m}$, with average rock quality designation (RQD) of $75 \%$, best matches the seismic data, and is closely correlated to the mine's borehole core observations. This finding has useful implications for efforts to remove tunnel reflections from seismograms recorded by standard (short borehole) geophones underground. As such, it is a necessary first step before standard microseismic monitoring arrays can be used to image the rock mass beneath and ahead of a working underground mine using reflection seismology.
\end{abstract}

Keywords: seismic reflection imaging, underground seismic exploration

\section{Introduction}

Reflection seismology is widely used for exploration in the oil and gas industry and often used in greenfields exploration for mining orebodies. Generally, the surveys use large-scale 2-D sensor arrays - sometimes up to 1,000 sensors - combined with active seismic sources, which are usually vibroseis trucks. While it's not possible to use similar sensor arrays and sources underground to explore around a working mine for additional orebodies, it would be very useful to apply some reflection seismology techniques in this setting. This current work aims to use a few standard triaxial geophones to image the surrounding rock mass for significant reflectors, in order to guide brownfields exploration activities from the underground mine.

There have been a few recent publications (Soma et al. 2007; Reshetnikov et al. 2015) based on seismogram data recorded by deep boreholes installed to monitor geothermal stimulations (hydraulic fracturing). The seismic events induced by the hydraulic pressure are used as sources of seismic radiation to image geological discontinuities in the vicinity of the seismicity. Reshetnikov et al. (2010) used geophones installed in deep boreholes to image reflectors in the vicinity of the San Andreas Fault in California, with natural microseismicity as the sources of seismic radiation.

The work by Soma et al. (2007) used reflected SS'-waves recorded at a few triaxial sensors and found significant reflectors at the Soultz geothermal site in France. These reflectors were consistent with geological interpretations of data recorded by a full surface seismic survey.

Reshetnikov et al. (2010) used reflected PP'-waves to produce images of strong reflectors near the San Andreas Fault which were consistent with previous imaging results and with the borehole core data.

Most recently, Reshetnikov et al. (2015) used both PP'- and SS'-reflections to image geological discontinuities at the Basel geothermal site in Switzerland. Their results were corroborated by ultrasonic logs and borehole core data. 
These publications are of interest as they use a few sparse triaxial geophones and passive seismic data to image the zone around the geophones and/or seismic events. Over 300 mines around the world routinely perform passive seismic monitoring of induced microseismicity using sparse triaxial geophone arrays. Thus these techniques are good candidates for application in underground mines.

\section{$2 \quad$ Reflected seismic waves}

When a seismic wave encounters a change of acoustic impedance, which is the product of density and seismic velocity, part of the seismic energy is reflected from that surface. The reflected energy is generally carried by both $\mathrm{P}$ and $\mathrm{S}$-waves, due to phase conversion at the interface for oblique intersection. If the incoming wave was a P-wave, then the reflected P-wave is denoted a PP' wave and the reflected S-wave is denoted PS'. Similarly, the waves reflected by an incoming S-wave are the SP' and SS'-waves. Figure 1 shows this diagrammatically.

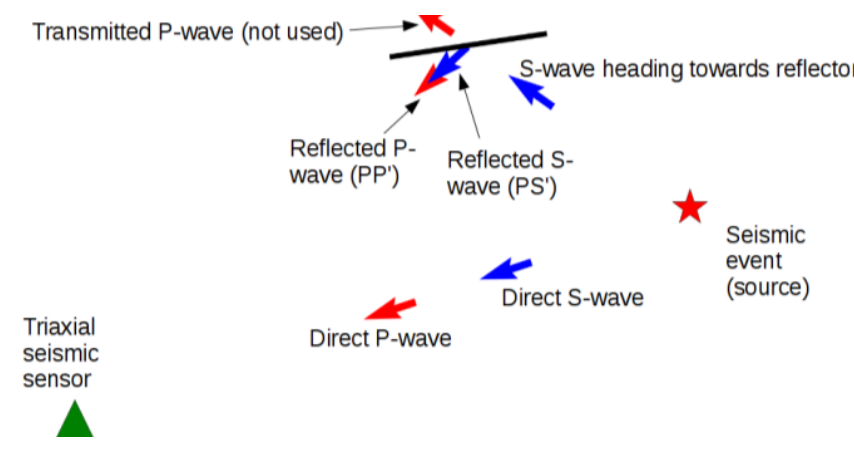

(a)

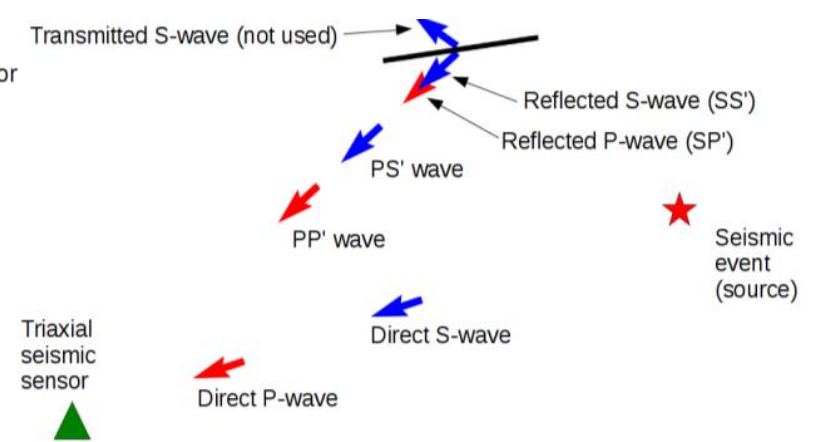

(b)

Figure 1 The $\mathrm{PP}^{\prime}$ and $\mathrm{PS}^{\prime}$-waves reflected after a $\mathrm{P}$-wave has arrived at the reflector (a); and the $\mathrm{SP}^{\prime}$ and $S^{\prime}$-waves reflected after the S-wave arrives at the reflector (b). Even in this trivial case, the seismogram would contain six arrivals: the two direct wave arrivals and the four reflected wave arrivals

P-waves are longitudinal waves, so the rock particles vibrate in the direction of travel. These waves are thus linearly polarised along the direction that the wavefront is travelling at the sensor position. In an isotropic and homogeneous medium, this direction is simply the vector from the last scatterer to the seismic sensor.

S-waves are shear waves, so the rock particles vibrate perpendicularly to the direction that the wavefront is travelling at the sensor position. In an isotropic medium, the S-wave is linearly polarised. If the medium is anisotropic, with different seismic velocities in different directions (for example a layered sedimentary rock), then the S-wave typically splits as it travels. At first, this splitting results in an increasingly elliptically polarised S-wave. Later, when the S-wave has split into two distinct waves, these S-wave groups will again be linearly polarised.

Generally, the host rock around hard rock mines is approximately isotropic, and we do not observe significant S-wave splitting for the reasonably short source-sensor distances that mine passive seismic monitoring considers.

If reflected waves arrive at a sensor during a 'quiet' period (i.e. a time distinct from the arrival times of other waves), that piece of the seismogram has a strongly polarised character. This can be used both to detect the arrival, as well as to limit the back propagation to directions consistent with the polarisation: along the polarisation direction for a P-wave and perpendicular to it for an S-wave.

\section{A method of imaging reflected seismic waves}

To estimate where the reflections are coming from, the time and polarisation of the reflected waves are used under the assumption that there has been a single scatterer. 
The polarisation directions are measured using a short time window of the triaxial seismogram and by performing a singular value decomposition, which yields three eigenvalues $e_{\mathrm{i}}$ and three corresponding eigenvectors $v_{i}$. The eigenvector corresponding to the dominant eigenvalue is the direction of polarisation, while the degree of polarisation (DoP) is obtained using the formula (Samson 1977):

$$
D o P=\frac{\left(e_{1}-e_{2}\right)^{2}+\left(e_{1}-e_{3}\right)^{2}+\left(e_{2}-e_{2}\right)^{2}}{2\left(e_{1}+e_{2}+e_{3}\right)^{2}}
$$

which varies between 1 for a perfectly linearly polarised signal and 0 for an unpolarised signal. The time window length is selected to include a few dominant periods of seismic wave.

To image the reflectors in 3D, while reducing the artefacts from the fact that only a few sensors are being used, the Fresnel volume migration method (Lüth et al. 2005; Buske et al. 2006) is employed. This is a simple extension of the standard Kirchhoff pre-stack depth migration, and takes into account the dominant frequency of the recorded wavelet and the fact that at finite frequencies the seismic ray is actually a tube.

The algorithm tries to use all four reflected waves: PP', PS', SP' and SS'. Often, the PS' and SP'-waves arrive close together and their polarisations are blurred. This sometimes happens too if the PP', PS' or SP'-waves arrive at the same time as the direct S-wave.

At each grid point on a regular 3D grid, the seismograms are analysed under the assumption that this grid point is a candidate reflector. If the point was indeed a reflector, then the reflected waves would arrive at a time calculated using the source-reflector and reflector-sensor distances together with the known seismic wave velocities for the homogeneous and isotropic rock mass. At those times in the seismograms, the DoP for the wavelets is calculated. If the DoP is higher than some threshold, the DoP is added to a cumulative DoP associated with that grid point.

After this procedure has been carried out for all grid points and all recorded seismograms, any significant reflectors emerge as points with high cumulative DoP.

To validate the algorithm, a synthetic test case was used. A 3D surface was used to separate two elastic materials with different acoustic impedance. A few triaxial seismic sensors were set in the model space and some seismic source positions chosen. The sensor positions, seismic source locations and geological contact surface were chosen realistically, based on the actual sensor array, geology and recorded microseismicity at an Australian mine. The model was used as input for a finite difference kinematic elastic waveform modelling code and synthetic triaxial seismograms calculated. Then these seismograms were used as input to the reflection imaging algorithm, to confirm that the 3D reflective surface was retrieved. Figure 2 shows the imaging output from this algorithm, confirming that the algorithm works as expected.

This reflector imaging method works well with seismograms from seismic events located far from mining excavations, recorded by triaxial seismic sensors installed into deep boreholes. If the sensors or sources are close to mining excavations, then strong reflections from the rock/air interface obscure the weak reflections from interesting geological discontinuities. While most seismic events locate close to the mining excavations that are inducing them, there are usually a few seismic events located far from excavations so this restriction is not a big problem. However, most standard mine seismic monitoring sensors are installed into short holes 5-10 $\mathrm{m}$ from the tunnels and so it would be convenient to use data from these sensors. In this case, which is unique to mining, the imaging procedure must be modified to disregard these strong reflections. Kinematic modelling can be performed using the known positions of the mining excavations, and this can possibly be used to remove the strong reflections from the seismograms. However, an investigation into the effects of the fracture zone surrounding the tunnels is required before this removal can be done accurately enough. To this end reflections from a known strong reflector - the shell of an open pit mine - were examined. 
6

9

5

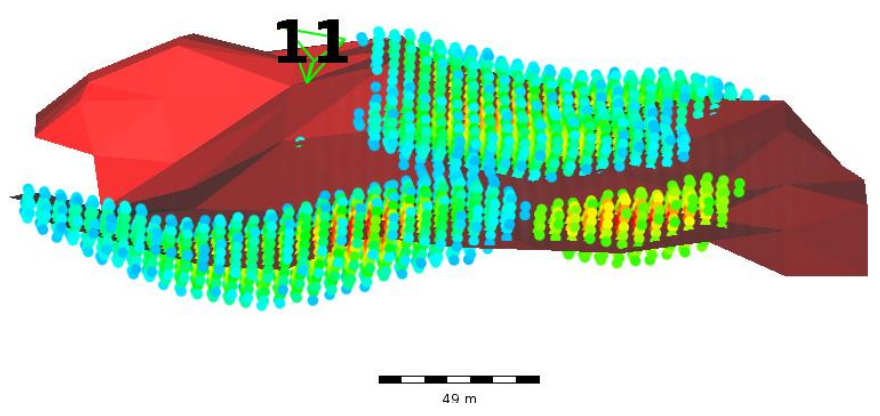

Figure 2 An example of imaging results. The reflector in this example (red surface) is used in the kinematic modelling to obtain synthetic seismograms at a few sensors (green triangles). The reflection imaging procedure with these seismograms then yields patches on this surface (coloured dots), confirming that the algorithm works as expected

\section{Reflections from the pit bottom}

To study the effects of the fracture zone around an air/rock reflector, seismogram data recorded by a deep borehole triaxial geophone installed beneath the open pit mine at the Williams mine in Hemlo, Canada was considered. At this mine both sensors and seismic events are located in the crown pillar between open pit mine and underground stopes (Figure 3).

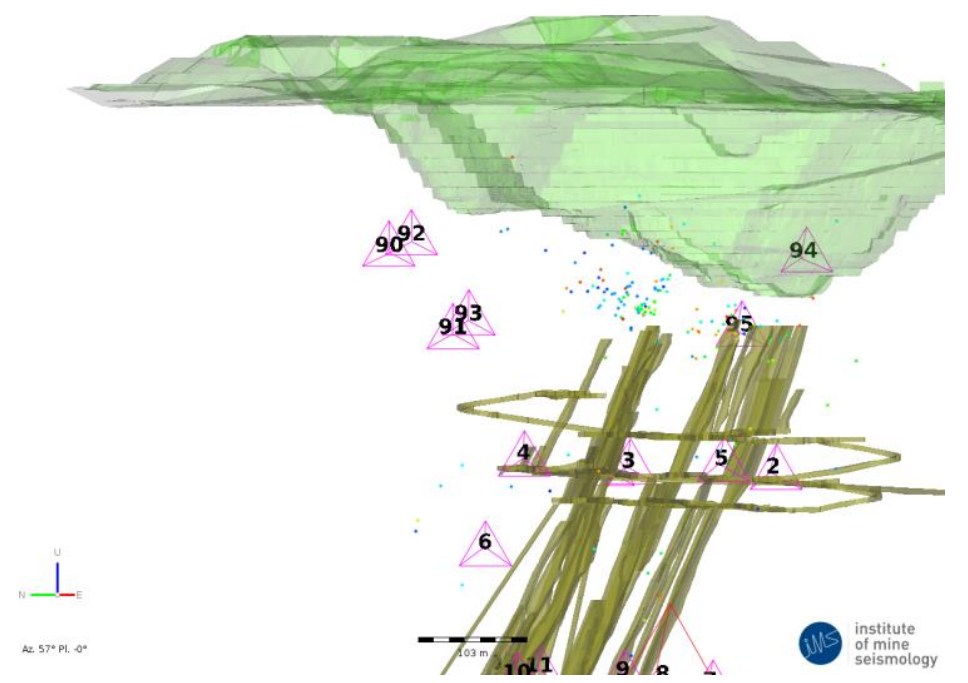

Figure 3 The Williams mine near Hemlo, Ontario, Canada. The Institute of Mine Seismology (IMS) microseismic monitoring system there includes some deep borehole triaxial geophones (purple triangles, 90-93) and recorded seismic events in the crown pillar (coloured dots). The reflections from the pit shell above (green) were used to study the effects of the fracture zone in this study

This study uses 405 events recorded between 1 February and 6 March 2016 and located in the crown pillar, with local magnitude ranging from -3.6 to -1.0. An analysis of the polar diagrams for the direct S-wave arrivals on these triaxial geophones reveals generally strong linearity, indicating an approximately isotropic rock mass. 
Seismic waves slow down in fractured rock. If the wavelength of the seismic wave is greater than the crack diameter, the cracks are dry and randomly oriented and the crack density, $e$, is sufficiently low such that they are non-interacting, the effective elastic properties of the rock mass is given by (Kuchanov 1992):

$$
E=E_{0}\left[1+\frac{16\left(1-v_{0}^{2}\right)\left(1-0.3 v_{0}\right)}{9\left(1-0.5 v_{0}\right)} e\right]^{-1}
$$

and

where:

$$
G=G_{0}\left[1+\frac{16\left(1-v_{0}\right)\left(1-0.2 v_{0}\right)}{9\left(1-0.5 v_{0}\right)} e\right]^{-1},
$$

$E \quad=$ effective Young's modulus.

$G \quad=$ effective shear modulus.

$E_{0}$ and $G_{0}=$ intact rock parameters.

$v_{0}=$ intact rock Poisson's ratio.

$e \quad=$ crack density defined by Bristow (1960):

$$
e=\frac{1}{V} \sum r_{i}^{3}
$$

where:

$r \quad=$ crack radius.

$V \quad=$ volume of rock being quantified.

From the effective moduli, the P-wave velocity, $v_{\mathrm{P}}$, and S-wave velocity, $v_{\mathrm{S}}$ can be easily shown using the standard equations (e.g. Aki \& Richards 2002) to be:

$$
v_{S}=\sqrt{\frac{G}{\rho}}
$$

and

$$
v_{P}=\sqrt{\frac{G(4 G-E)}{\rho(3 G-E)}}
$$

Combining Equations (2), (3), (5) and (6) yields the effective seismic body-wave velocities as a function of the crack density $e$. The work of Grechka and Kuchanov (2006) has shown that Equations (2) and (3) are valid even well into the domain of interacting cracks, which will be the case in the fractured zone around the open pit.

The analysis of the reflections from the pit focused on the SS' reflection and took the following form:

- Choose a likely effective thickness for the fracture zone surrounding the pit $-20 \mathrm{~m}$ seemed to be a plausible value after discussions with the mine geotechnical personnel.

- For every trial value for the crack density:

- For every seismic event in our dataset:

- Estimate the reflected wave path from source to deep borehole sensors 90 and 91 via the pit shell, assuming the minimum linear path length.

- Compute the expected travel time for the SS' reflection, taking into account the seismic travel time delay as a result of travel through the fracture zone, reflection off the pit surface and travel back out of the fracture zone, using Equations (2), (3), (5) and (6).

- Examine the piece of seismogram at the expected SS' time. Is there a clear polarisation at this time?

- If there is a clear polarisation, does the polarisation vector normal include the postulated pit shell reflection point? Yes or No? 
- Calculate the score: $\mathrm{N}_{Y} /\left(\mathrm{N}_{\mathrm{Y}}+\mathrm{N}_{\mathrm{N}}\right)$, which is the fraction of distinct arrivals with polarisation directions consistent with a pit reflection. This score would be around $9 \%$ for random polarisations - the area of the Fresnel zone over the total spherical area.

An example of one of the analysed seismograms is shown in Figure 4. A DoP threshold of 0.7 was used. Apart from the strong polarisations present when the direct $\mathrm{P}$ and $\mathrm{S}$-waves arrive, a significant wavelet of coherent polarisation is apparent in the coda.

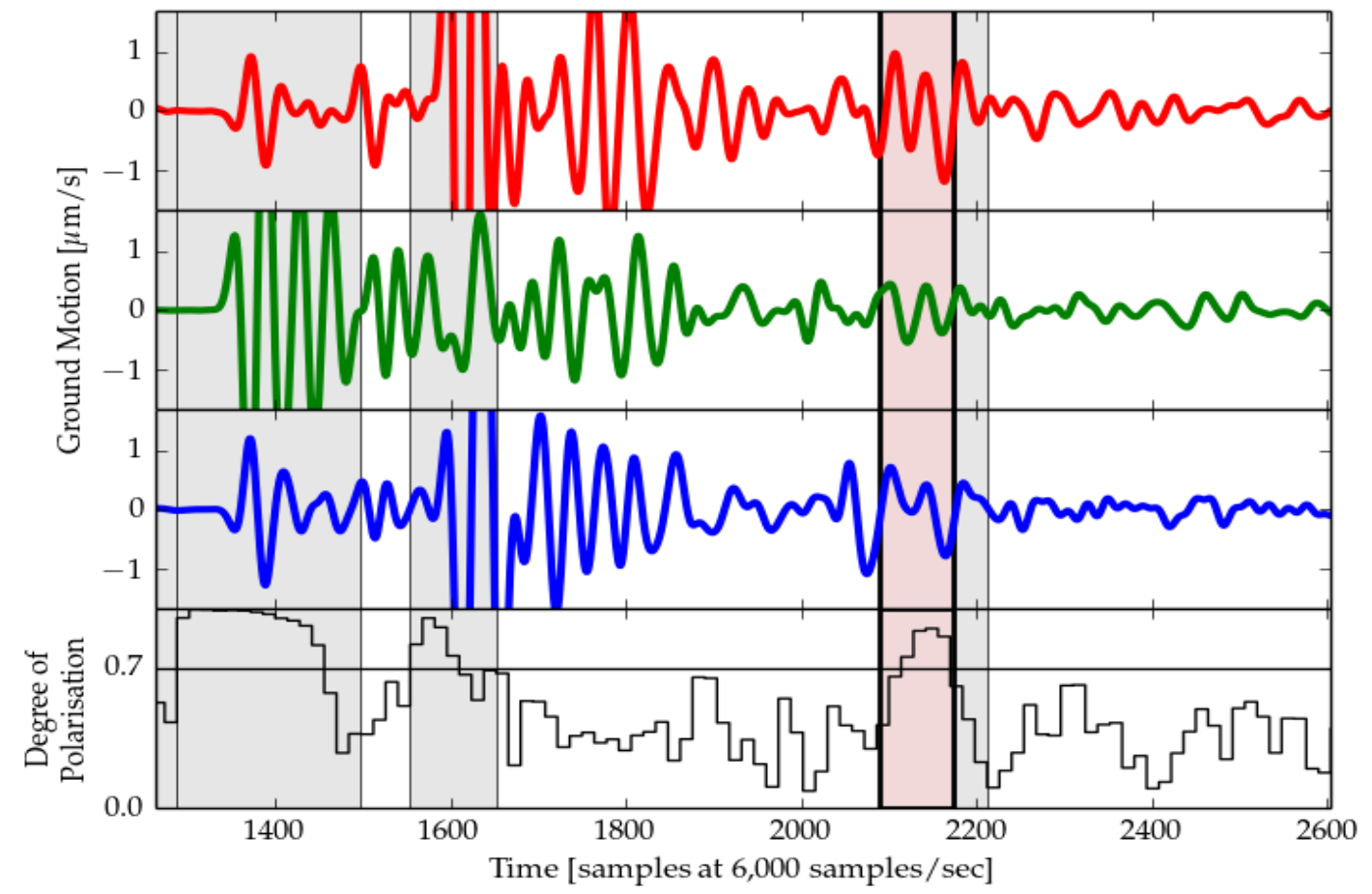

Figure 4 An example of the Williams mine triaxial seismograms (top three waveforms) with the Degree of Polarisation (DoP) shown on the bottom graph. Apart from the direct $\mathrm{P}$ - and S-wave arrivals, the DoP indicates the arrival of a reflected wavelet after sample 2,100 (pink shading)

A graph of score versus crack density is shown in Figure 5 . There is a maximum score at a crack density of between 1.42 and 1.51 . The maximum score is $50 \%$, which is much higher than the expected $9 \%$ score for random polarisations. Note that for a zero crack density (neglecting the fracture zone) the score is a rather modest $12 \%$, confirming that the fracture zone cannot be ignored for reflection work!

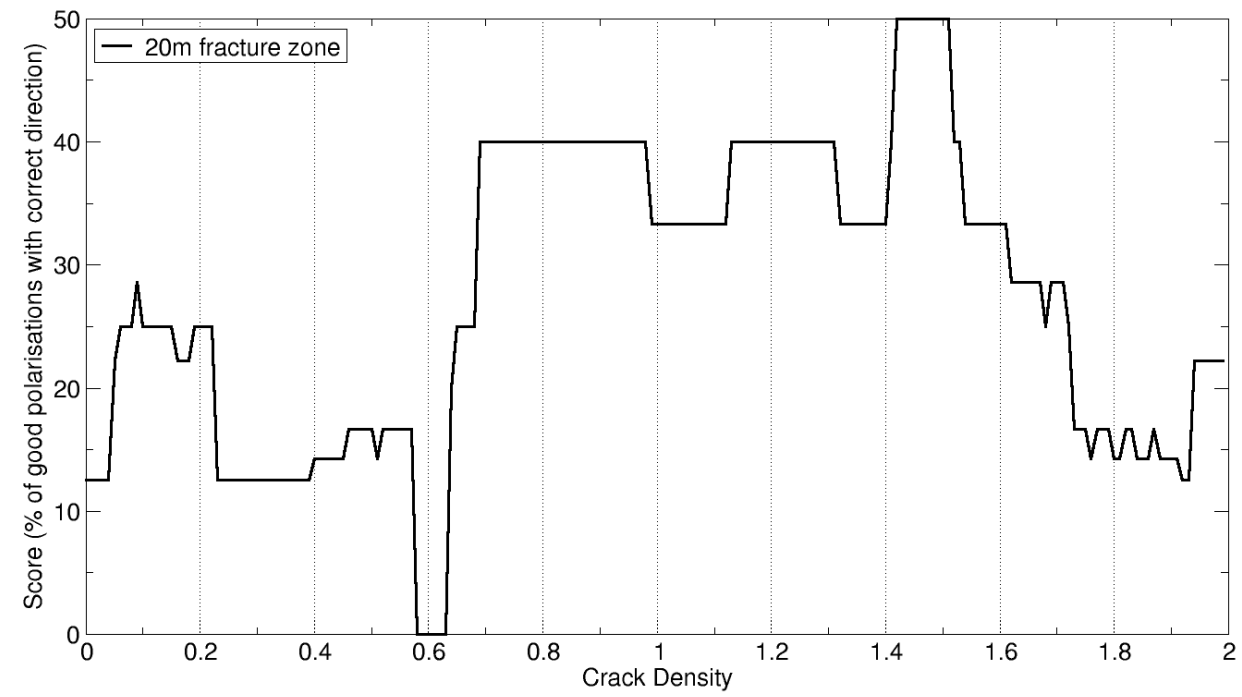

Figure 5 Results of the pit reflection analysis. The maximum score corresponds to an indicated fracture zone average crack density of between 1.42 and 1.51 
Crack density is not a parameter used commonly in mine geotechnical engineering, and so we cannot easily see if a crack density of 1.5 is reasonable for the open pit fracture zone. The author is not aware of a theoretical relation between crack density and the popular RQD (Deere 1964) measure, so a small numerical simulation was performed. A $3 \times 3 \mathrm{~m}^{2}$ volume of rock was populated with randomly oriented circular cracks with diameter chosen from a uniform distribution up to a maximum diameter of 0.2-0.4 $\mathrm{m}$. The crack density is calculated and then RQD is measured using a virtual NX-diameter core. The results from this simulation are shown in Figure 6: a crack density of around 1.5 corresponds to an RQD of around $75-80 \%$.
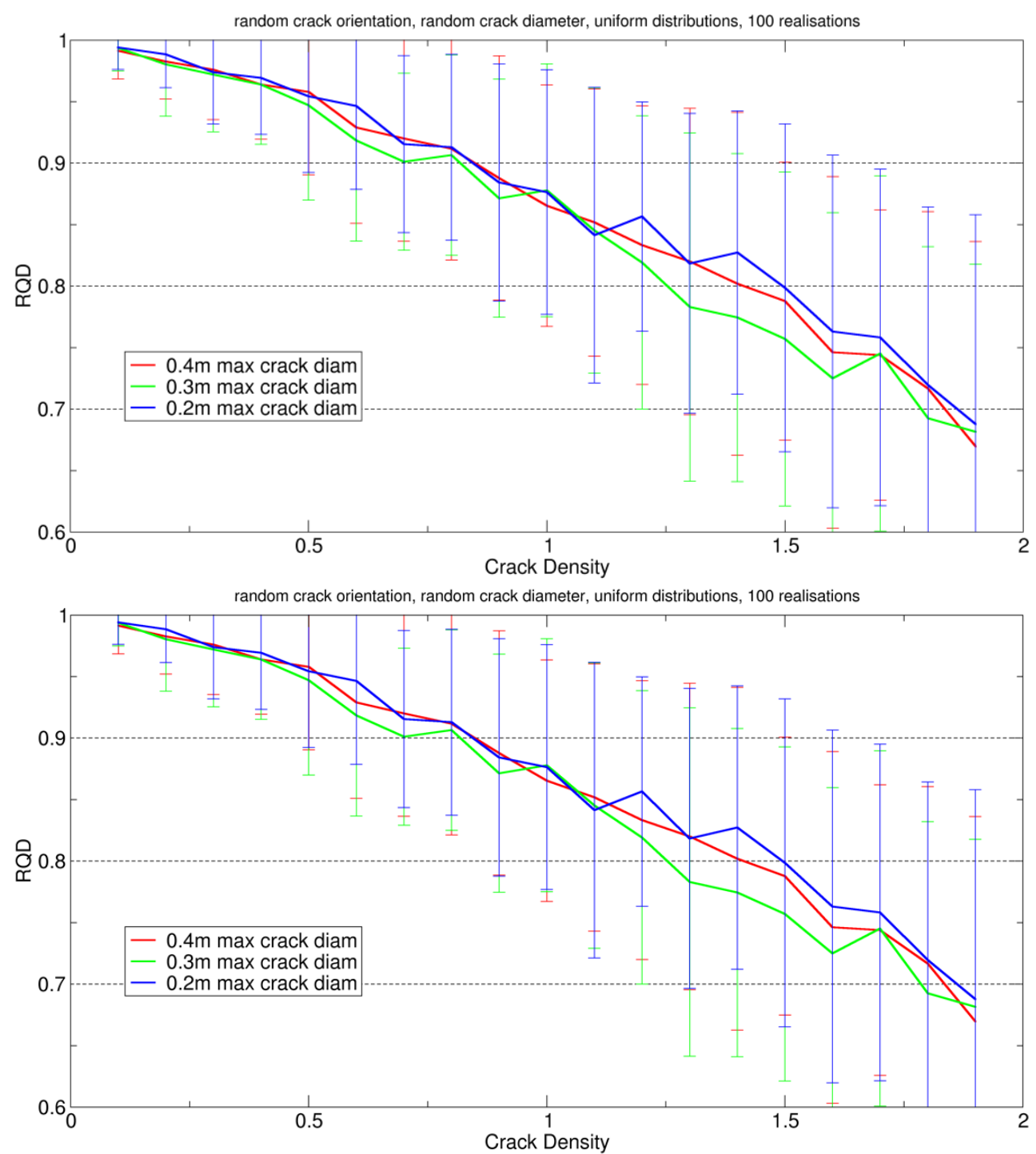

Figure 6 The measured relationship between RQD and crack density, based on a rock mass populated with randomly oriented cracks of uniformly distributed diameter up to a maximum crack diameter of $0.2,0.3$ and $0.4 \mathrm{~m}$. Notwithstanding the large 1-sigma error bars, a crack density of 1.5 corresponds to an RQD of $75-80 \%$

Thus the seismological analysis of reflected waves from the Williams mine pit shell would suggest an RQD of $75-80 \%$ averaged over a $20 \mathrm{~m}$ thick fracture zone. This finding agrees nicely with borehole core data from the mine (Figure 7). 


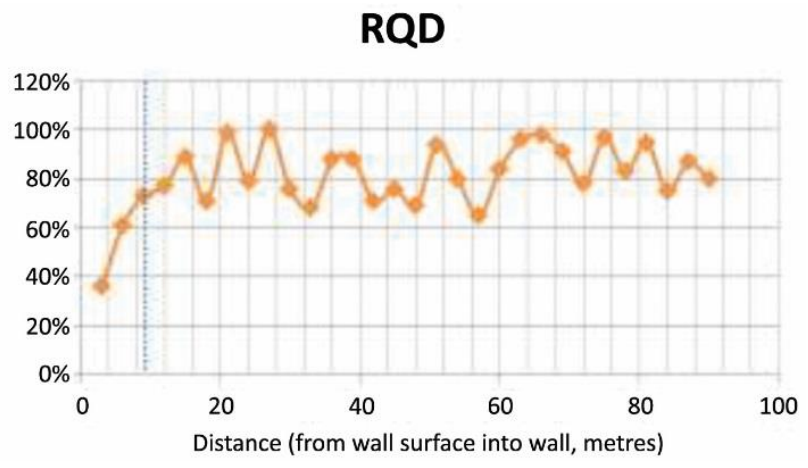

Figure 7 RQD versus distance from pit wall surface, measured with NX core at the Williams mine open pit

\section{Conclusion}

Sparse sensor reflection seismology, using the Fresnel volume migration technique, is a promising new tool for guiding underground exploration and reducing drilling costs. Normal mining-induced microseismic events are used as sources of seismic radiation, and standard geophones are used to record the seismograms. Currently, the method requires both deep borehole $(>200 \mathrm{~m})$ geophones and seismic events located far $(>200 \mathrm{~m})$ from excavations. In that special case, the imaging algorithm needs only slight adaptation from those used in geothermal projects and thus can be used today.

However, it would be advantageous if standard short borehole geophones and normal seismic events could also be used to image geological discontinuities around the working mine. For that, we need to understand the effects of the fracture zone surrounding underground excavations. This study showed how seismic data does indeed contain information about some of the properties of the fracture zone, and confirmed that the fracture zone cannot be ignored for such reflection work. This finding represents an important step toward developing a more general seismic reflection imaging algorithm for underground mines.

\section{Acknowledgement}

This work was supported by the IMS Research Patrons - Anglogold-Ashanti, AngloPlatinum, Codelco (El Teniente), Goldfields (South Deep), Harmony Gold, LKAB, Newcrest Mining and Northam Platinum. I also thank Mr Cullen Fleming at Williams mine for assistance, permission to use this data and advice, as well as Barrick Gold (Williams mine) for permission to publish this work.

\section{References}

Aki, K \& Richards, PG 2002, Quantitative Seismology, University Science Books, California.

Bristow, JR 1960, 'Microcracks and the static and dynamic elastic constants of annealed and heavily cold-worked metals', British Journal of Applied Physics, vol. 11, pp. 81-85.

Buske, S, Heigel, M \& Lüth, S 2006, 'Fresnel-volume-migration of single-component seismic data', Proceedings of the 68th Annual Conference and Exhibition, Extended Abstracts, European Association of Geologists and Engineers, G044.

Deere, DU 1964, 'Technical description of rock cores', Rock Mechanics Engineering Geology, vol. 1, pp. 16-22.

Grechka, V \& Kuchanov, M 2006, 'Effective elasticity of fractured rocks - A snapshot of the work in progress', Geophysics, vol. 71, no. 6, pp. W45-W58.

Kuchanov, M 1992, 'Effective elastic properties of cracked solids: Critical review of some basic concepts', Applied Mechanics Reviews, vol. 45 , no. 8, pp. 304-335.

Lüth, S, Buske, S, Gortz, A \& Giese, R 2005, 'Fresnel-volume-migration of multicomponent data', Geophysics, vol. 70, no. 6, pp. S121-S129. Reshetnikov, A, Buske, S \& Shapiro, SA 2010, 'Seismic imaging using microseismic events: results from the San Andreas fault system at SAFOD', Journal of Geophysical Research, vol. 115, B12324.

Reshetnikov, A, Kummerow, J, Asanuma, H, Haring, M \& Shapiro, SA 2015, 'Microseismic reflection imaging and its application to the basel geothermal reservoir', Geophysics, vol. 80, pp. WC39-49.

Samson, JC 1977, 'Matrix and stokes vector representations of detectors for polarized waveforms: Theory, with some applications to teleseismic waves', Geophysical Journal of the Royal Astronomical Society, vol. 94, pp. 1817-1830.

Soma, N, Niitsuma, H \& Baria, R 2007, 'Reflection imaging of deep reservoir structure based on three-dimensional hodogram analysis of multicomponent microseismic waveforms', Journal of Geophysical Research, vol. 112, B11303. 